\title{
Photovoice-Forschung mit Jugendlichen - ein Leitfaden zur Durchführung
}

\section{Sandra Layh, Anja Feldhorst, Rebecca Althaus, Monika Bradna und Petra Wihofszky'}

\section{Zusammenfassung}

In diesem Leitfaden wird die Anwendung der partizipativen Forschungsmethode Photovoice praxisnah vorgestellt. Anhand des Beispiels einer gemeinsamen Forschung von Jugendlichen, Akteur*innen des Sozial- und Gesundheitswesens und Wissenschaftler*innen werden die einzelnen Schritte dieser partizipativen Forschungsmethode illustriert. Von der Planung und Vorbereitung über die Durchführung bis hin zur Evaluation kann so nachvollzogen werden, was in den jeweiligen Phasen von Photovoice zu beachten ist und wie die konkrete Umsetzung von Projekten aussehen kann. Praktiker*innen finden im Leitfaden eine Anleitung für ihre eigenen Photovoice-Vorhaben.

1 Sandra Layh | Hochschule Esslingen | Sandra.Layh@hs-esslingen.de

Anja Feldhorst | Freie Lektorin und Autorin | info@lektorat-feldhorst.de

Rebecca Althaus | Hochschule Esslingen | RebeccaAlthaus@stud.hs-esslingen.de

Monika Bradna | Stabsstelle Sozialplanung der Stadt Esslingen | monika.bradna@ esslingen.de

Petra Wihofszky | Hochschule Esslingen | Petra.Wihofszky@hs-esslingen.de

(C) Der/die Autor(en) 2020

S. Hartung et al. (Hrsg.), Partizipative Forschung, https://doi.org/10.1007/978-3-658-30361-7_8 


\section{Schlüsselbegriffe}

Photovoice; Partizipation; partizipative Forschung; qualitative Methoden; Jugendliche

\section{Einführung}

Mit der partizipativen Forschungsmethode ${ }^{2}$ Photovoice können Sie gemeinsam mit Jugendlichen Bedarfe für Angebote und Maßnahmen erforschen und Empfehlungen entwickeln. Unter einer vorher festgelegten Fragestellung gehen die Jugendlichen ins Feld, fotografieren und werten die Fotos anschließend gemeinsam aus. Die Jugendlichen dokumentieren und reflektieren Missstände und Stärken in ihren Lebenswelten, arbeiten eigene Anliegen heraus, um all dies an Entscheidungsträger^innen zu vermitteln und so Veränderungen in Gang zu bringen.

Photovoice wird international eingesetzt, zum Beispiel in den USA von Brustkrebspatientinnen, die eine bessere Versorgung erreichen wollen, oder in Deutschland von afrikanischen Communities in Hamburg, die - im Rahmen des DAH-Projekts „PaKoMi“ - mit Photovoice ihren HIV-Präventionsbedarf bestimmt haben (www. pakomi.de, siehe Anhang in diesem Beitrag sowie von Unger \& Gangarova 2011).

Sie selbst organisieren den Rahmen für die Photovoice-Forschung, schulen die Jugendlichen zu ethischen und rechtlichen Fragen der Lebenswelt-Fotografie, zu den Inhalten des Forschungsthemas sowie ggf. zur Technik und begleiten den Forschungsprozess während der Datensammlung, -diskussion und -auswertung.

Der vorliegende Leitfaden vermittelt Ihnen praxisnah, wie Sie vorgehen können und was dabei zu beachten ist.

Wir stellen Ihnen die sieben Schritte einer Photovoice-Studie vor und illustrieren sie anhand des gemeinsamen Photovoice-Projekts der Hochschule Esslingen und der Stadt Esslingen. Unsere Esslinger Photovoice-Studie fand innerhalb des Forschungsprojekts „KEG - Kommunale Entwicklung von Gesundheitsstrategien: Wissenschaft und Praxis im Dialog" statt (partkommplus.de, siehe Anhang in diesem Beitrag). Wir erforschten gesundheitliche Vorstellungen, Interessen und Anliegen von und mit Jugendlichen. Analog zu Jugendprojekten im internationalen Kontext

2 Begriffsbestimmung, Prinzipien und Merkmale partizipativer Forschung können Sie dem Beitrag 1 Partizipative Forschung - ein Forschungsansatz für Gesundheit und seine Methoden in diesem Buch entnehmen. 
wollten wir die Gesundheit in jugendlichen Lebenswelten fördern und Bedingungen für die aktive Beteiligung von Jugendlichen schaffen. Die Studie wurde in einem Bezirk mit einer sehr jungen Wohnbevölkerung durchgeführt. Ein Großteil der Kinder und Jugendlichen dort ist von Armut betroffen oder bedroht.

Hintergrundinformationen zu Photovoice, die ausführliche Darstellung der KEG-Studie und weitere Praxisbeispiele finden Sie in Beitrag 4 Photovoice als partizipative Methode: Wirkungen auf individueller, gemeinschaftlicher und gesellschaftlicher Ebene in diesem Buch.

\section{Schritt 1 Die Photovoice-Forschung planen und vorbereiten}

\section{Eine Forschungsgemeinschaft einrichten}

Am Beginn einer Photovoice-Forschung steht die Einrichtung einer Forschungsgemeinschaft (sofern Sie das Projekt nicht allein in Angriff nehmen wollen). Dazu bringen Sie Personen an einen Tisch, die an der Lebenswelt ${ }^{3}$ Jugendlicher interessiert sind. Diese Gruppe plant die Photovoice-Forschung gemeinsam und setzt sie auch zusammen um. Laden Sie am besten Akteur ${ }^{\star}$ innen ein, die Entscheidungsbefugnisse im zu beforschenden Setting haben. Sie können selbstverständlich auch interessierte Jugendliche und allgemein interessierte Personen in die Forschungsgemeinschaft einbeziehen.

Die Forschungsgemeinschaft trifft sich über den gesamten Projektzeitraum hinweg. Je nach Ausrichtung und Umfang der Photovoice-Forschung sowie den zeitlichen Kapazitäten der Beteiligten kann dies wöchentlich oder auch in größeren Intervallen sein. Ob die gesamte Forschungsgemeinschaft an allen Schritten der Photovoice-Forschung beteiligt ist oder ob Sie arbeitsteilig vorgehen, ist abhängig davon, wie Sie deren Rolle definieren.

Im KEG gehörten zur Forschungsgemeinschaft jeweils ein bis zwei Akteur*innen aus:

- Jugendhaus

- Gemeinwesenarbeit

3 Lebenswelt und Setting werden hier synonym verwendet. Es handelt sich dabei um soziale Systeme, in denen Menschen unter gegebenen Verhältnissen und Bedingungen leben, lernen, arbeiten, spielen usw. (siehe Hartung \& Rosenbrock 2015). 
- Kirchengemeinde

- Familienzentrum

- Sportvereinen

- Erziehungshilfe

- Grünflächenamt

- Jugendhilfeplanung

- Ordnungsamt

- Amt für Soziales und Sport

- Volkshochschule

- Hochschule

Insgesamt waren wir 15 Personen.

Jugendliche stießen erst zu einem späteren Zeitpunkt zur Forschungsgemeinschaft, da sich die Idee, mit der Methode Photovoice zu forschen und Jugendliche als Mitforschende einzubeziehen, erst im Prozess mit den beteiligten Akteur ${ }^{\star}$ innen entwickelte.

Unsere Forschungsgemeinschaft traf sich im ca. zwei Jahre dauernden Forschungszeitraum durchschnittlich jeden zweiten Monat. Die Treffen dauerten zwischen zwei und drei Stunden.

\section{Ziele, Fragen und Forschungsfeld in der Forschungsgemeinschaft abstimmen}

In der Forschungsgemeinschaft handeln Sie gemeinsam aus, welche Ziele Sie mit der Photovoice-Forschung verfolgen, welche Forschungsfragen Sie hierzu beantworten, das heißt erforschen, und wo Sie forschen wollen.

In dieser Phase lernen sich die Beteiligten kennen, bauen gegenseitiges Vertrauen auf und entwickeln (und verwerfen) viele Ideen. Eine gemeinsame Arbeitsbasis entsteht.

Geben Sie der Forschungsgemeinschaft Raum und Zeit, Themen aufkommen zu lassen, Aspekte zu benennen, die die Beteiligten interessieren und beschäftigen, Wünsche, aber auch Einwände und Bedenken offen zu äußern. Dann haben Sie eine Basis, um die Schwerpunkte zu bestimmen, Forschungsziele und -fragen zu entwickeln.

Im KEG benötigte die Formulierung von Zielen und Forschungsfragen ein halbes Jahr mit fünf Treffen. Zunächst wurden Themen gesammelt. In Kleingruppen fand anschließend ein Austausch entlang folgender Fragen statt: 
- Was soll in der Fallstudie ins Zentrum gestellt werden, sodass die Teilnehmer*innen einen Gewinn für die Arbeit mit Jugendlichen haben?

- Was sind aus Sicht derjenigen, die mit dieser Zielgruppe arbeiten, Anliegen im Stadtteil?

Die Ergebnisse des Austauschs wurden zusammengetragen und ein Schwerpunkt mit seinen verschiedenen Facetten herausgearbeitet. Auf dieser Basis formulierten wir handlungsleitende Ziele und Forschungsfragen:

\section{Ziele}

- Gesundheit in jugendlichen Lebenswelten alltagsnah fördern

- (Rahmen-)Bedingungen für die Beteiligung von Jugendlichen schaffen

- Jugendliche zur eigenständigen Gestaltung ihres Bezirks anregen

\section{Fragen}

- Welche gesundheitlichen Anliegen und Interessen haben die Jugendlichen des Bezirks?

- Wo sehen sie welche Handlungsbedarfe, um sich im Bezirk gesund und wohl fühlen zu können, und welche Ideen haben sie, diese zu verwirklichen?

Im KEG wurde als Forschungsfeld ein Bezirk mit einem hohen Anteil an Kindern und Jugendlichen gewählt, die von Armut betroffen oder bedroht sind.

Für die spätere Forschungsphase mit den Jugendlichen formulieren Sie die Forschungsfragen so, dass die Jugendlichen Fotos dazu machen können.

Wie wir im KEG unsere Forschungsfragen in bearbeitbare Fragen „übersetzt“ haben, können Sie unter Schritt 2 nachlesen.

\section{Option: Aufgabenprofil und Honorar für eine Begleitperson bestimmen}

Finden Sie ggf. eine Person, die die forschenden Jugendlichen in der Photovoice-Forschung begleitet und unterstützt. Das kann sinnvoll sein, wenn Sie mit jüngeren Jugendlichen oder Kindern arbeiten, die Aufsicht oder Unterstützung benötigen. Oder wenn sich die Forschung über mehrere Termine, einen längeren Zeitraum oder ein großes Gebiet erstreckt, sodass die Organisation aufwendig und die gesammelten Daten umfangreich werden. 
Legen Sie die Aufgaben und, sofern vorgesehen, das Honorar fest, bevor Sie sich auf die Suche nach einer geeigneten Person aus der Forschungsgemeinschaft oder von außerhalb machen.

Im KEG gehörte die Begleitperson nicht zur Forschungsgemeinschaft, wurde aber über ein Mitglied der Forschungsgemeinschaft vermittelt. Die Begleitperson hatte kurz vor Beginn des Photovoice-Projekts ihr Freiwilliges Soziales Jahr im Jugendhaus abgeschlossen. Daher kannte ein Großteil der mitforschenden Jugendlichen diese Person bereits und vertraute ihr.

Die Begleitperson wirkte an den Workshops mit den Jugendlichen mit, begleitete die Jugendlichen während ihrer Forschungstätigkeit, hielt sowohl Kontakt zu den Jugendlichen als auch zu uns, sammelte alle an die Jugendlichen ausgehändigten Unterlagen sowie deren Fotos und übermittelte sie an uns.

\section{Mitforschende finden}

Nachdem die Rahmenbedingungen geklärt sind, gilt es, Jugendliche als Mitforschende für die Photovoice-Forschung zu gewinnen.

Sinnvoll ist es, wenn diejenigen Akteur*innen der Forschungsgemeinschaft, die im Forschungsfeld arbeiten, Kontakt zu den Jugendlichen aufnehmen.

Folgende Tipps können Ihnen helfen, Jugendliche für Ihr Photovoice-Projekt $\mathrm{zu}$ interessieren:

- Sprechen Sie die Jugendlichen direkt und persönlich an.

- Klären Sie Ziele, Fragen und Rahmenbedingungen der Forschung im Gespräch.

- Sprechen Sie die Jugendlichen als Expert*innen ihrer Lebenswelt an.

- Kommunizieren Sie den Mitbestimmungsspielraum in der Forschung offen.

- Beziffern Sie die Zahlung einer etwaigen Aufwandsentschädigung. ${ }^{4}$

- Stellen Sie Verbindlichkeit her, indem Sie zum Beispiel im Gespräch mit den Jugendlichen ein Datum für eine Rückmeldung festlegen.

- Lassen Sie Ihre Kontaktdaten da, damit die Jugendlichen ggf. zu einem späteren Zeitpunkt Fragen an Sie richten können.

4 Ob Sie eine solche bezahlen oder anderweitige Anreize für die Mitwirkung gewähren, ist abhängig vom Forschungssetting und den beteiligten Jugendlichen. In den USA, wo sehr viel mit partizipativen Methoden wie Photovoice geforscht wird, ist es z. B. üblich, die Mitforschenden für ihre Mitwirkung zu entlohnen. 
Im KEG wurden Jugendliche von Akteur ${ }^{\star}$ innen der Forschungsgemeinschaft angesprochen. So ging beispielsweise der Leiter des Jugendhauses persönlich auf jugendliche Besucher*innen zu, der evangelische Pfarrer im Bezirk auf ehemalige Konfirmandinnen. Wir fanden insgesamt neun Jugendliche, die sich als Mitforschende an unserer Studie beteiligten.

Wir zahlten den Jugendlichen für ihre Mitwirkung ein Honorar. Bei jedem Treffen spendierten wir außerdem für alle Pizza.

\section{Material für die Photovice-Forschung erstellen}

Bevor Sie gemeinsam mit den Jugendlichen in die praktische Phase der Photovoice-Forschung einsteigen können, benötigen Sie verschiedene Dokumente, zum Beispiel um die Teilnahme festzuschreiben, die Jugendlichen ausreichend zu informieren usw.

Folgende Dokumente können notwendig sein:

- Informationsschreiben über das Vorgehen, die Ziele und Rahmenbedingungen des Photovoice-Projekts

- Teilnahmevereinbarung

- Einverständniserklärung zur Verwendung und Verarbeitung der erhobenen Daten

- weiteres Informationsmaterial mit zum Beispiel einer Terminübersicht, den Kontaktdaten, dem vereinbarten Ablauf und den Deadlines, den Forschungsfragen, den Regeln für das Fotografieren usw.

Besprechen Sie in der Forschungsgemeinschaft, welche Dokumente in Ihrer Forschung gebraucht werden.

Im KEG wurden eine Teilnahmevereinbarung für die Mitwirkung der Jugendlichen, eine Einverständniserklärung zur Verwendung der Daten der Photovoice-Forschung, ein allgemeines Informationsblatt über die Photovoice-Forschung sowie ein Handout mit allen Terminen und Kontaktdaten der Begleitperson erstellt. Ein weiteres mit den Fragen, zu denen die Jugendlichen Fotos erstellten, kam später hinzu.

\section{Schritt 2 Die Jugendlichen zu Mitforschenden schulen}

Wenn Sie die Planung und Vorbereitung der Photovoice-Forschung abgeschlossen haben, schulen Sie die Jugendlichen zu Mitforschenden. Durch die Schulung(en) 
lernen sich die Jugendlichen gegenseitig kennen, werden mit dem Thema der Forschung vertraut und lernen, was die Methode Photovoice ist und wie sie diese anwenden können. Die Inhalte lassen sich gut erfahrungsorientiert umsetzen.

Integrieren Sie unbedingt Einheiten zu Datenschutz und ethischen Fragen in die Schulung. Denn diese Aspekte sind für die Arbeit mit Fotografien besonders wichtig. Zum Beispiel ist bei Aufnahmen von Personen in fast allen Fällen deren Einverständnis notwendig. Je nach Alter und Kenntnisstand der Jugendlichen bieten sich zusätzlich praktische Übungen zum Fotografieren und Auswählen von Motiven an.

Sie können sich bei der Konzeption Ihrer Schulung(en) gerne an dem exemplarischen Ablauf orientieren, wie wir ihn nachfolgend für die KEG-Schulung beschreiben.

Im KEG hatten wir für die Schulung ein Treffen mit einer Dauer von fünf Stunden einschließlich Pausen vereinbart. Durchgeführt wurde das Treffen von den Wissenschaftler*innen der Hochschule, der Kooperationspartner ${ }^{\star}$ in der Stadt sowie der Begleitperson. Zusätzlich hatten wir einen externen Experten eingeladen, der Teile der Schulung filmte und fotografierte und die Jugendlichen zum Thema Fotografieren schulte.

\section{Beispiel für den Ablauf einer Schulung im KEG}

\section{Was wurde benötigt?}

- Flipchart mit Tagesablauf

- 2 Pinnwände

- Moderationskoffer

- Seil, Tau oder Ähnliches

- Klebestreifen für Namensschilder

- TN-Liste (Name, E-Mail, Adresse, Termin, Unterschrift)

- Verpflegung

\section{Begrüßung und Vorstellung des Tagesablaufs}

Wir begrüßten alle Jugendlichen und stellten uns und den externen Experten vor. Wir holten das Einverständnis ein, Fotos und Filmaufnahmen während der Schulung machen zu dürfen, um ggf. später gemeinsam aus diesen Aufnahmen einen Film über das Projekt erstellen zu können (zum Film an späterer Stelle mehr).

Wir beantworteten Fragen zum Projekt, die in den Anwerbungsgesprächen mit den Akteur*innen offen geblieben waren, und stellten den geplanten Ablauf für 
die Schulung vor. Außerdem notierten wir für das spätere gemeinsame Essen die Pizzawünsche der Jugendlichen.

\section{Soziometrische Aufstellungen zum Kennenlernen und Einstieg in das Thema Gesundheit}

Damit wir die Jugendlichen besser kennenlernten und sie sich untereinander auch, stellten wir vier Kennenlernfragen. Bei der ersten Frage baten wir die Jugendlichen, sich entsprechend aufzustellen:

- In welchem Teil von Bezirk wohnt ihr? (Ecken des Raumes für die Stadtteile des Bezirks)

Bei den nächsten drei Fragen gaben wir nicht vor, wie sich die Jugendlichen positionieren sollten, sondern überließen ihnen die Entscheidung:

- Wer kennt wen?

- Wer macht was am liebsten?

- Wer von euch isst was am liebsten?

Zum Abschluss der Kennenlernphase legten wir ein Seil auf den Boden. Wir stellten die Frage: „Wie geht es dir heute? Wie fühlst du dich?“ und baten die Jugendlichen, sich entlang des Seils zu positionieren. Ein Ende stand dabei für „Es geht mir sehr gut“, das andere Ende für „Es geht mir nicht gut“.

\section{Erfahrungsorientierter Austausch über Wohlbefinden/Gesundheit}

Wir nutzten die Seilübung als Überleitung zum Erfahrungsteil und zum Austausch über Gesundheit. Wir fragten:

- Wo und warum hast du dich hier eingeordnet?

- Wovon hängt es ab, wo du dich einordnest?

- Was bedeutet es für dich, dass du dich hier einordnest?

Die Jugendlichen teilten ihre Gedanken zu den Fragen mit. Durch weitere situationsbezogene, anregende Fragen initiierten wir einen Austausch über Gesundheit und Wohlbefinden. Die Antworten und Kommentare der Jugendlichen ordneten wir thematisch an der Pinnwand. So entstand ein interaktives Mindmap. 


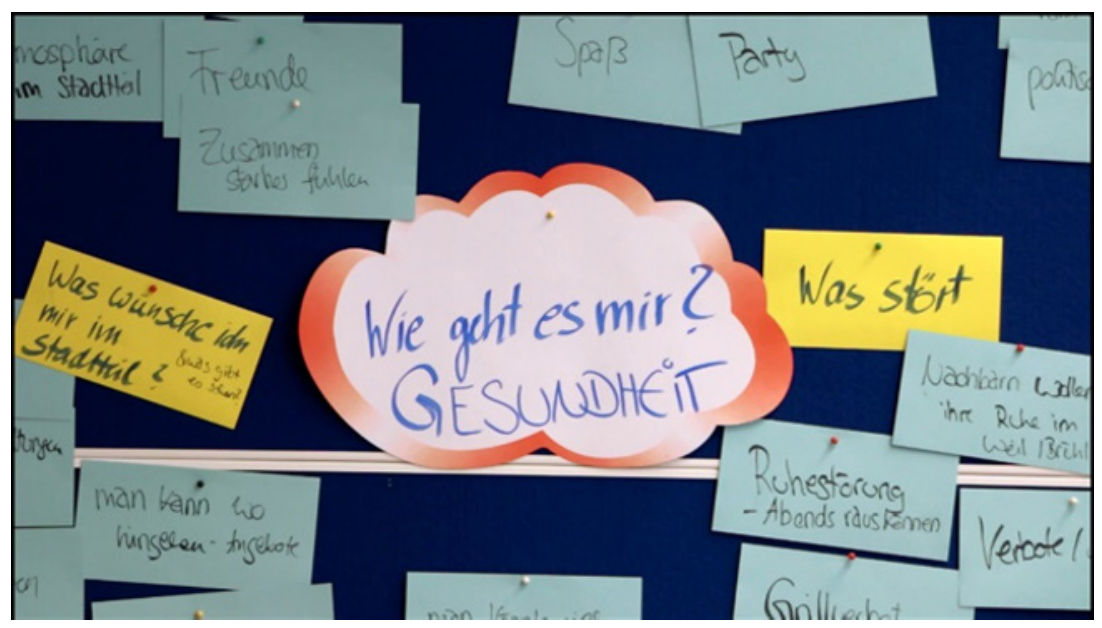

Abb. 1 Interaktives Mindmap (im Schulungsworkshop entstandenes Foto)

\section{Vorstellung der Methode Photovoice und gemeinsame Planung der Umsetzung}

Wir stellten die Grundlagen von Photovoice vor: zu ausgewählten Fragen Fotos machen, die Fotos miteinander diskutieren, die wichtigsten Themen gemeinsam herausarbeiten und auf deren Basis Empfehlungen dazu entwickeln, was sich wie ändern soll. Wir klärten, ob die Jugendlichen sich vorstellen konnten, so zu arbeiten, und ob jede ${ }^{\star}$ eine Kamera oder ein Handy mit eingebauter Kamera zur Verfügung hatte.

Dann erarbeiteten wir im Austausch mit den Jugendlichen ethische und rechtliche Aspekte des Filmens und Fotografierens. Der externe Experte zeigte ein Video und diskutierte mit den Jugendlichen die wichtigsten Punkte, die es beim Fotografieren zu beachten gilt:

- Bevor jemand gefilmt wird, muss diese Person gefragt werden.

- Die Person, die aufgenommen werden soll, muss immer freiwillig zustimmen.

- Die Person, die fotografiert werden soll, muss über den Zweck und die Verwendung der Fotos und Filmaufnahmen informiert werden.

- Gebäude dürfen nur von außen fotografiert werden, für Innenaufnahmen muss wieder um Erlaubnis gefragt werden.

- Auf den Fotos dürfen keine Dinge oder Szenen abgebildet sein, die den dort Abgebildeten in irgendeiner Form schaden könnten. 
Da die Jugendlichen bereits im Alltag viel mit ihren Handys fotografierten und wir außerdem Wert auf authentische Fotos legten, verzichteten wir auf Übungen zum Fotografieren.

Nun stellten wir die Forschungsfragen vor. Wir hatten die Forschungsfragen so umformuliert und aufbereitet, dass die Jugendlichen dazu Fotos machen konnten:

- Was mache ich gern?

- Was mag ich gar nicht?

- Wann geht's mir gut?

- Wann nicht?

- Wo halte ich mich gern auf?

- Und wo nicht?

- Was tut mir gut?

- Was tut mir nicht gut?

- Was wünsche ich mir?

In einem Brainstorming einigten wir uns auf die zu bearbeitenden Fragen und sammelten erste Ideen, wie welche Themen auf einem Foto oder anderweitig dargestellt werden könnten. Wir beschlossen gemeinsam mit den Jugendlichen, dass zu jeder Frage ein Foto und zu einer Frage ein Video gemacht würde. Wir besprachen abschließend Organisatorisches wie den Zeitraum für Fotoaufnahmen, die Termine für die Treffen mit der Begleitperson und den Austausch der Fotografien.

\section{Ausklang}

Beim gemeinsamen Essen klärten wir weitere Fragen zum Projekt und zur Umsetzung, stellten vor, wie es nach der Datenerhebung weitergehen würde (siehe nächste Schritte), händigten die erstellten Unterlagen aus und beantworteten Fragen zu diesen Unterlagen.

\section{Schritt 3 Fotografieren - Feldphase}

Sie vereinbaren mit den mitforschenden Jugendlichen einen Zeitraum, in dem geforscht wird. Die Jugendlichen machen mit ihren Handys, mit eigenen Kameras oder mit für die Photovoice-Forschung ausgehändigten (Einweg-)Kameras Fotos und Videos zu den gemeinsam festgelegten Fragen. 
Nach der Feldphase übermitteln die Jugendlichen ihre Aufnahmen, zugeordnet zu den jeweiligen Fragen, an Sie oder, falls Sie eine bestimmt haben, die Begleitperson.

Sie oder die Begleitperson speichern die Fotos so ab, dass sie den einzelnen Mitforschenden und der jeweiligen Frage zugeordnet werden können. Sie oder die Begleitperson bringen die Aufnahmen - am besten auf einem USB-Stick - zur Gruppendiskussion mit, dem nächsten Schritt der Photovoice-Forschung.

Im KEG hatten die Jugendlichen die Sommerferien über Zeit, ihre Fotos und Videos zu machen. Sie gingen zum Teil allein, zum Teil zu mehreren los. Auch trafen sie sich zu einem vereinbarten Termin mit der Begleitperson im örtlichen Jugendhaus, um auf einer Karte des Stadtteils mit Nadeln in verschiedenen Farben diejenigen Orte zu markieren, die sie gerne aufsuchen, und die, die sie nicht gern mögen. Über diese Markierungen kamen sie mit der Begleitperson ins Gespräch über diese Orte und entwickelten Ideen, was sie zu den Fragen fotografieren könnten.

Gegen Ende der Sommerferien trafen sich die Jugendlichen erneut mit der Begleitperson. Zu diesem Treffen brachten sie ihre Fotos und Videos auf einem Stick mit. Wurden mehrere Fotos zu einer Frage gemacht, suchten die Jugendlichen, zum Teil in Eigenregie, zum Teil gemeinsam mit der Begleitperson, das Foto aus, das die Frage aus ihrer Sicht am passendsten beantwortete.

Die Begleitperson brachte alle gesammelten Fotos in nach den Jugendlichen benannten Ordnern auf einem USB-Stick zur Gruppendiskussion (siehe nächster Schritt) mit.

\section{Schritt 4 In der Gruppe diskutieren}

In einem Workshop diskutieren Sie mit den Jugendlichen die Fotos und Videos, die diese in der Feldphase erstellt haben. Denn die Fotos sprechen nicht für sich, erst im Gespräch werden die jeweiligen Bedeutungen und die enthaltenen Themen erkennbar.

Viele Projekte orientieren sich in der Gruppendiskussion an den so genannten SHOWED-Fragen (siehe Beitrag 4 Photovoice als partizipative Methode: Wirkungen auf individueller, gemeinschaftlicher und gesellschaftlicher Ebene, Abschnitt 2, in diesem Buch sowie Wang 1999).

Für die späteren Schritte von Photovoice ist es entscheidend, dass Sie die Gruppendiskussionen auf Audio oder Video aufzeichnen oder sie anderweitig, zum Beispiel durch Notizen, dokumentieren. Diese Aufzeichnungen bilden die Grundlage für die Datenaufbereitung und -auswertung und die Ableitung von Handlungsempfehlungen. 
Statt Diskussion und Auswertung zu trennen, können Sie beide Schritte aber auch in einem Workshop durchführen. Viele Photovoice-Projekte machen das so. Vor allem solche, an denen keinen Wissenschaftler*innen beteiligt sind. (Die Wissenschaftler ${ }^{*}$ innen widmen sich meist in einem gesonderten Arbeitsschritt der Aufbereitung und Auswertung der Daten.) Die Erfinderinnen der Methode, Caroline C. Wang und Mary Ann Burris (1997), schlagen für diesen Fall vor, bereits die Auswahl der zu diskutierenden Fotos als ersten Auswertungsschritt zu sehen, die Erzählungen zu den jeweiligen Fotos in der bzw. den Gruppe(n) als zweiten und die anschließende gemeinsame Identifikation der enthaltenen wichtigsten Themen, bezogen auf die Forschungsfragen, als letzten Schritt in der Auswertung. Wenn Sie so vorgehen wollen, ist die Dokumentation der einzelnen Schritte noch wichtiger, damit Sie aus den Ergebnissen Handlungsempfehlungen ableiten können.

Im KEG waren Wissenschaftler*innen einer Hochschule beteiligt. Um eine tiefer gehende Auswertung zu ermöglichen und um die Ergebnisse wissenschaftlich zu fundieren, haben wir einen separaten, von den Wissenschaftler*innen übernommenen Auswertungsschritt in die Photovoice-Forschung integriert, einen zusätzlichen Auswertungsworkshop mit den mitforschenden Jugendlichen durchgeführt sowie die Ableitung von Handlungsempfehlungen in mehreren Terminen gemeinsam mit den Jugendlichen in der Forschungsgemeinschaft vollzogen (siehe die folgenden Schritte). Im KEG setzten wir daher für die Gruppendiskussion lediglich ein Treffen mit einer Dauer von fünf Stunden an. Durchgeführt wurde es von den Wissenschaftler*innen der Hochschule, der Kooperationspartner ${ }^{\star}$ in der Stadt sowie der Begleitperson.

\section{Beispiel für den Ablauf der Gruppendiskussion im KEG}

\section{Was wurde benötigt?}

- Räumlichkeit mit voneinander getrennten Zimmern

- Flipchart mit Tagesablauf

- Ausdrucke DIN A3 mit Fragen für die Gruppendiskussion

- Laptops (Anzahl je nach Gruppengröße)

- ggf. Beamer

- Aufnahmegerät(e) oder Videokamera(s)

- Klebestreifen für Namensschilder

- TN-Liste (Name, E-Mail, Adresse, Termin, Unterschrift)

- Verpflegung 


\section{Begrüßung und Vorstellung des Tagesablaufs}

Wir begrüßten die Jugendlichen und stellten den geplanten Tagesablauf sowie den Zweck dieses Treffens vor. Wir klärten organisatorische Fragen und holten noch ausstehende Unterlagen ein, zum Beispiel fehlende unterzeichnete Einverständniserklärungen.

\section{Feedback-Runde}

Nach der Begrüßung starteten wir mit einer Feedbackrunde. Wir hatten die Jugendlichen während der Feldphase in den Sommerferien nicht gesehen und wollten daher Folgendes von ihnen und auch von der Begleitperson wissen:

- Wie ist es gelaufen beim Fotografieren? Wie ging es euch mit dieser Aufgabe?

- Was war einfach?

- Was war vielleicht auch schwierig?

- Was hat euch überrascht?

Wir hielten die Rückmeldungen der Jugendlichen und der Begleitperson schriftlich fest, um sie für die Evaluation (siehe letzter Schritt der Photovoice-Forschung) nutzen zu können.

\section{Gruppendiskussion mit je vier bis fünf Jugendlichen und einem*einer Moderator*in}

Die Jugendlichen bildeten nun zwei Gruppen mit je vier und fünf Personen. Wer mit wem in eine Gruppe ging, war ihnen überlassen.

Jede Gruppe nahm einen Laptop und suchte sich einen eigenen Raum. Für die Diskussion der acht Fotos und des einen Videos pro Person legten wir einen ungefähren Richtwert von 45 Minuten fest. Die Moderation innerhalb der Gruppe übernahm eine Person von uns.

Vor Beginn der Gruppendiskussionen holte der*die jeweilige Moderator*in das Einverständnis ein, das Gespräch auf Tonband aufzuzeichnen. Er*sie erklärte, dass es geplant sei, die Tonbandaufnahmen abzutippen und die so entstehenden Texte auszuwerten.

Zu Beginn der Diskussion erhielten die Teilnehmenden die Anweisung: Erst spricht die Gruppe, dann der*die Fotograf*in/Filmer ${ }^{\star}$ in. Wir wollten so freies Assoziieren und die gemeinsame Reflexion ermöglichen. Ausführlicher erläutern wir die Gründe für unser beschriebenes Vorgehen in unserem Beitrag 4 Photovoice als partizipative Methode: Wirkungen auf individueller, gemeinschaftlicher und gesellschaftlicher Ebene in diesem Buch. 
Die Bilder und Videos wurden nun pro Person und Frage gemeinsam auf dem Laptop angesehen.

Frage an die Gruppe:

- Was seht ihr?

Fragen an den*die Fotograf*in/Filmer*in:

- Wie kam es dazu, dass du das zu dieser Frage fotografiert hast?

- Wofür steht das Foto für dich?

Frage an die Gruppe:

- Was bedeutet das für euch Jugendliche im Bezirk?

\section{Feedbackrunde zur Gruppendiskussion}

Nachdem die Gruppen von ihren Diskussionen zurückgekehrt waren, führten wir eine weitere Feedbackrunde durch, diesmal zu den Erfahrungen und Themen in den Gruppendiskussionen, und fragten:

- Wie ging es euch in der Diskussion? Wie war es, eure Fotos und Videos zu zeigen und gemeinsam zu besprechen?

- Was war besonders eindrücklich? Warum?

- Gab es gemeinsame Themen?

- Gab es auch ganz unterschiedliche Themen? Oder unterschiedliche Ansichten?

Auch dieses Feedback notierten wir, um es in die Auswertung und die Evaluation (siehe Schritt 7) einzubeziehen. Diese Rückmeldung gab Aufschluss über die den Jugendlichen wirklich wichtigen Themen, denn wir gingen davon aus, dass sie speziell diese erinnerten und daher an dieser Stelle benannten.

\section{Ausklang}

Beim Ausklang klärten wir offene Fragen und stellten vor, was mit den aufgenommenen Gruppendiskussionen passieren würde, bis wir uns zum Auswertungsworkshop wiedersähen (siehe nächster Schritt). Noch einmal sahen wir die kurzen Videos der Jugendlichen an und beschlossen gemeinsam, aus diesen sowie den Filmaufnahmen der Schulung und den Fotos der Jugendlichen einen Film 
zu erstellen. Hierfür holten wir gleich das Einverständnis der Jugendlichen ein. Diesen Film wollten wir unmittelbar nach der Auswertung angehen, um mit ihm die wesentlichen Belange und Themen der Jugendlichen anschaulich an Entscheidungsträger ${ }^{\star}$ innen zu transportieren.

Unser fertiges Video können Sie sich ansehen unter: http://partkommplus.de/ teilprojekte/keg/wie-stellen-sich-jugendliche-ihren-stadtteil-vor/. Wie es entstanden ist, können Sie unter Schritt 6 nachlesen.

\section{Zwischenschritt Die Diskussionen aufbereiten}

Dass Photovoice-Projekte gemeinsam von Praktiker*innen und Wissenschaftler*innen durchgeführt werden, ist eher die Ausnahme als die Regel.

Wenn Sie Ihr Projekt ohne wissenschaftliche Partner*innen machen, ist es sinnvoll, dass Sie das Material aus der Feldphase und den Gruppendiskussionen für den nächsten Schritt, die partizipative Auswertung und Formulierung von Handlungsempfehlungen, aufbereiten. Es sei denn, Sie haben bereits im Rahmen der Gruppendiskussion die Auswertung durchgeführt (siehe Schritt 4). Dennoch werden Sie vermutlich ein weiteres Treffen mit den Jugendlichen benötigen, um die herausgefilterten Themen gemeinsam zu bestätigen, ggf. anzureichern und Handlungsempfehlungen abzuleiten.

Es geht bei der Aufbereitung nicht darum, nach streng wissenschaftlichen Standards das Material zu analysieren - auch wenn wir das im KEG getan haben (siehe unten). KEG stellt eher die Ausnahme dar. Bei Ihrer Datenaufbereitung steht vielmehr das Reduzieren des Materials im Mittelpunkt, damit die anschließende Entwicklung von Handlungsempfehlungen mit den Jugendlichen und der Forschungsgemeinschaft zielgerichtet verlaufen kann. Fassen Sie die vorhandenen Fotos, Videos (bzw. Aussagen aus Videos) und Themen zu Gruppen zusammen, wählen Sie Beispielfotos aus und ergänzen Sie diese, wenn möglich, mit prägnanten Aussagen der Jugendlichen aus der Diskussion. Überlegen Sie sich, wie Sie die Themen anschaulich präsentieren können. Denn Sie sollten gemeinsam mit den Jugendlichen überprüfen, ob wirklich alle wichtigen Themen erfasst wurden oder ob etwas ergänzt werden sollte. Ein Beispiel für die Präsentation der Ergebnisse finden Sie im Schritt 5.

So können Sie das umfangreiche Material reduzieren und die relevanten Themen herausfiltern: Gehen Sie die Tonband- oder Videoaufnahmen von den Gruppendiskussionen in Ruhe, ggf. auch mehrmals durch und notieren die darin vorkommenden Themen. Behalten Sie dabei immer die Forschungsfrage(n) im 
Hinterkopf. Liegen Ihnen abgetippte Versionen der Gruppendiskussionen vor, so können Sie mit unterschiedlichen Farben für die verschiedenen Themen arbeiten.

Sie stellen in Ihrer Bearbeitung des Materials sicher fest, dass gewisse Themen wiederkehren und sich verschiedene Aussagen der Jugendlichen unter ein Überthema fassen lassen. Bestimmt sind solche Zusammenhänge bereits in der Gruppendiskussion von den Jugendlichen selbst festgestellt worden. Achten Sie daher auf Aussagen wie: „....und was damit zusammenhängt...., „...das finde ich auch und außerdem...“, ,...dazu gehört auch noch...“, ,... ich bin ganz deiner/eurer Meinung und..." usw. So entsteht nach und nach eine Liste mit Themen und Unterthemen, die Sie bei dem nächsten Termin mit den Jugendlichen gemeinsam prüfen können.

Wenn Sie Ihr Photovoice-Forschungsprojekt zusammen mit Wissenschaftler*innen durchführen, so werden diese in der Regel die Aufbereitung der Gruppendiskussionen übernehmen. Wie diese Aufbereitung konkret erfolgt, ist abhängig vom Ziel und den Fragen der Photovoice-Forschung.

Die Wissenschaftler*innen der Forschungsgemeinschaft im KEG bereiteten die Daten (Fotos und Videos, Aufnahmen der Gruppendiskussionen) folgendermaßen für einen Auswertungsworkshop mit den Jugendlichen auf:

Die auf Tonband aufgezeichneten Gruppendiskussionen wurden wörtlich transkribiert.

Die so entstandenen Texte wurden in ein elektronisches Auswertungsprogramm geladen und dort mehrere Male gelesen. Die verschiedenen Themen wurden bei jedem Durchlauf markiert und mit einem Titel versehen. Mit jedem weiteren Durchlesen wurden die Benennungen verfeinert, übergeordnete Themen erkannt und Facetten herausgearbeitet. Dieser Schritt ist vergleichbar mit dem so genannten „offenen Codieren“ nach Barney Glaser und Anselm Strauss (1998), die die „Grounded Theory" zur Auswertung qualitativer Daten entwickelt haben.

Anschließend wurden die Fotos bzw. Videos, durch die die jeweiligen Themen in die Diskussion gebracht worden waren, den codierten Textstellen zugeordnet.

Im nächsten Schritt, der Feinanalyse, prüften wir für jede Textstelle, was die Jugendlichen wahrgenommen und wie sie es bewertet hatten. So wurden zum Beispiel manche Punkte als störend, andere als gut, schön usw. bewertet. In einem zweiten Analyseschritt arbeiteten wir heraus, wie die Jugendlichen sich das Thema aneignen, welche Bedeutung es für sie hat. Also aus welchen Gründen es sie zum Beispiel stört, dass Orte ungepflegt sind. Es existiert zum Beispiel ein Platz direkt am Fluss, an dem die Jugendlichen sich gerne aufhalten, Sport treiben und sich mit Freunden zum Quatschen, Relaxen und Abschalten treffen. Er wurde einerseits als schön, ruhig und perfekt zum Chillen beschrieben, andererseits aber auch als heruntergekommen und ungepflegt. Man könne sich deshalb dort „nicht so richtig wohlfühlen"(Zitat eines Mitforschenden). In diesen Aussagen zeigte sich das An- 
liegen der Jugendlichen, diesen Ort für sich nutzen zu wollen, dies aber nicht oder nur mit Einschränkungen tun zu können.

Zumeist benannten die Jugendlichen in den Gruppendiskussionen bereits konkrete Handlungsbedarfe für die verschiedenen Themen, die von uns in einem letzten Analyseschritt herausgearbeitet wurden. So wurde für die Stelle am Fluss vorgeschlagen, die Bänke zu erneuern und Straßenlaternen zu installieren. Außerdem wünschten sich die Jugendlichen dort Mülleimer oder einen Hinweis, dass man seinen Müll wieder mitnehmen solle.

Für diesen Auswertungsschritt nutzten wir auch das Feedback, das die Jugendlichen nach der Gruppendiskussion gaben. So konnten wir prüfen, ob wir in unserer Auswertung alle Themen berücksichtigt hatten, die die Jugendlichen nach den Diskussionen als relevant erwähnten.

Nun glichen wir die Ergebnisse der bisherigen Analyseschritte ab und entfernten alle Doppelungen. So konnten wir auf einen Blick sehen,

- was Jugendliche in ihrem Bezirk wahrnehmen und was ihre Beurteilung dazu ist,

- was Jugendliche in ihrem Bezirk gerne machen (wollen) sowie

- welche Veränderungen den Jugendlichen wichtig sind.

Aus diesem Ergebnis ermittelten wir die Veränderungsbedarfe im Bezirk aus der Sicht der Jugendlichen:

- Stadtteil pflegen

- Müll beseitigen

- Orte jugendgerecht gestalten

- Orte nutzen dürfen

- Neue Orte für Jugendliche schaffen

- Aktivitäten für Jugendliche anbieten

- Verkehrsbedingte Missstände ändern

- Vorurteile ausräumen

Auf diesen Handlungsbedarfen lag der Fokus für die weitere Arbeit mit den Jugendlichen.

Als letzten Schritt bereiteten wir die Präsentation der herausgearbeiteten Handlungsbedarfe vor, um im Auswertungsworkshop mit den Jugendlichen zu prüfen, ob wir alle wichtigen Themen erfasst hatten. Wie die Ergebnispräsentation für den Workshop aussah, können Sie im Schritt 5 sehen. 


\section{Schritt 5 Partizipativ auswerten und Handlungsempfehlungen erarbeiten}

Zu Beginn der Auswertung stellen Sie den mitforschenden Jugendlichen die aufbereiteten Ergebnisse vor. Sie holen von den Jugendlichen Feedback ein, um herauszufinden, ob die Themen, die den Jugendlichen wichtig sind, erfasst wurden. Sie können die Ergebnisse zusammen mit den Jugendlichen ergänzen, anreichern, priorisieren und ggf. lokalisieren.

Falls die Mitglieder der Forschungsgemeinschaft an den vorangegangenen Workshops mit den Jugendlichen nicht beteiligt waren, präsentieren Sie auch diesen zunächst die Ergebnisse. Die Jugendlichen erarbeiten dann gemeinsam mit den Mitgliedern der Forschungsgemeinschaft, was wo wie passieren kann und soll. Diese Handlungsempfehlungen werden im nächsten Schritt umgesetzt oder an zuständige Stellen weitergetragen. Je nach Schwerpunkt und Fragen Ihrer Photovoice-Forschung steht hier bereits der 6. Schritt an, die Ergebnisse auf geeignete Art zu veröffentlichen. In der Regel verläuft der Übergang von der Formulierung von Handlungsempfehlungen zur Veröffentlichung der Ergebnisse sowieso fließend, da das Ziel nahezu jeder Photovoice-Forschung Veränderungen sind, die ohne die Bekanntmachung der Ergebnisse meist nicht zu erreichen wären.

Im KEG wurden die Auswertung und die Ableitung von Handlungsempfehlungen in separaten Treffen realisiert. Am ersten Treffen, einem fünfstündigen Auswertungsworkshop mit den Jugendlichen, nahm nicht die gesamte Forschungsgemeinschaft teil. Nach dem Auswertungsworkshop mit den Jugendlichen gab es zwei Treffen mit den Jugendlichen und der gesamten Forschungsgemeinschaft. Dort wurden die Ergebnisse der Jugendlichen diskutiert und gemeinsam Handlungsvorschläge sowie Lösungsmöglichkeiten erarbeitet.

Beispiel für den Ablauf eines Auswertungsworkshops mit den jugendlichen Mitforschenden im KEG:

\section{Was wurde benötigt?}

- Flipchart mit Tagesablauf

- große Pinnwand mit Stadtteilkarte

- Moderationskoffer

- Klebepunkte

- Moderationskarten mit den Themen der Jugendlichen

- Laptop zur Präsentation der Datenaufbereitung

- Beamer

- Klebestreifen für Namensschilder 
- TN-Liste (Name, E-Mail, Adresse, Termin, Unterschrift)

- Verpflegung

\section{Begrüßung und Vorstellung des Tagesablaufs}

Wir begrüßten die Jugendlichen und stellten den geplanten Tagesablauf sowie den Zweck dieses Treffens vor.

\section{Ergebnisse und Vorgehensweise der Datenaufbereitung vorstellen}

Wir erklärten den Jugendlichen zunächst, wie wir von den Tonbandaufnahmen der Gruppendiskussionen und den diskutierten Fotos und Videos zu den Ergebnissen der Datenaufbereitung gekommen waren. Dann präsentierten wir am Beamer zunächst die Ergebnisse im Überblick, um dann nacheinander immer ein Ergebnis mit je einem exemplarischen Foto oder Videoausschnitt und einer markanten Textstelle aus den Gruppendiskussionen genauer zu verdeutlichen. Alle weiteren Aspekte zum Thema, die durch das exemplarische Foto und die Textpassage nicht abgedeckt waren, zeigten wir als stichwortartige Aufzählung. Zum Thema „Stadtteil pflegen“" zum Beispiel sah das Ganze so aus:

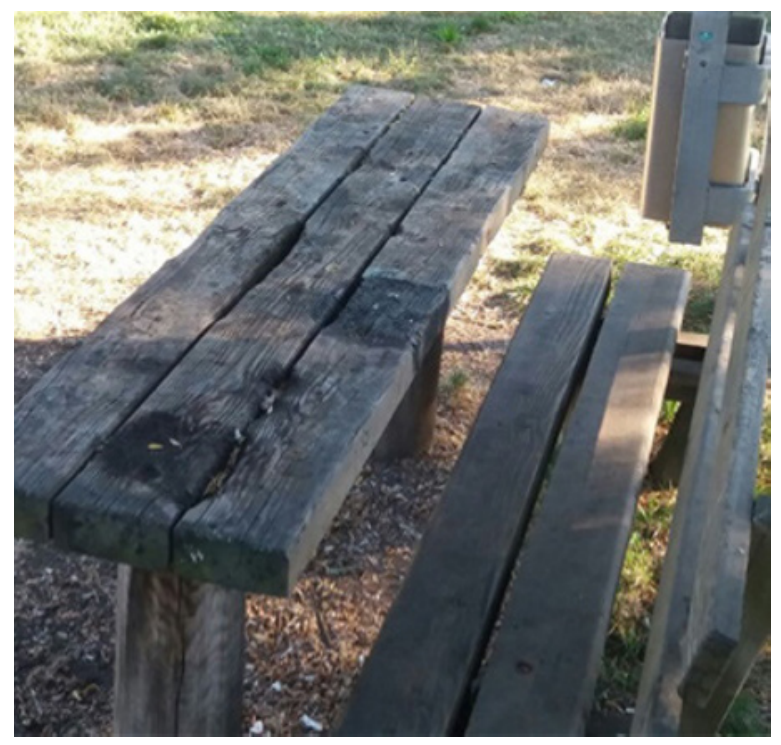

Abb. 2 Stadtteil pflegen (Exemplarisches Foto eines mitforschenden Jugendlichen) 


\section{Abb. 3 \\ Exemplarische Textpassage aus der Gruppendiskussion:}

B: Ja wie gesagt man könnte statt Sand Rindenmulch, also man könnte das ersetzen, dann den Asphalt noch putzen, weil das ist wirklich extrem rutschig und vielleicht halt noch so diese Wippen und so befestigen oder überhaupt neu machen und vielleicht noch eine große Rutsche für Kinder. Ja also, man könnte schon so Einiges machen. Und mehr Sitzbänke, da gibt es nur sehr wenig Sitzbänke.

A: Einfach regelmäßig den Spielplatz pflegen, irgend so ein Putzdienst kommt. $D$ : Auch mal so kontrollieren, ob irgendwas locker ist oder so, weil da steht jetzt, wie ich es gehört habe, diese Wippe, da war ich lange nicht mehr drauf, aber wo ich mal war, früher war es schon stabiler und jetzt nicht mehr und das muss man schon kontrollieren, regelmäßig.

Was gehört noch dazu:

- Unterführung Bahnhof: Graffitis entfernen, streichen, putzen, renovieren

- Aktivspielplatz: Bänke erneuern, Unebenheiten auf vorhandenem Fußballplatz beseitigen, regelmäßiger Rasen mähen

- Hartplatz: Schmierereien entfernen, Mülleimer regelmäßiger leeren, Linien nachziehen

- Straßen im Bezirk: Schlaglöcher ausbessern

- Bahnhofspielplatz: Glasscherben beseitigen, Geräte kontrollieren/sanieren

- Fußballplatz am Einkaufszentrum: Rasen mähen, insgesamt instandhalten

\section{Themen prüfen und priorisieren}

Nachdem wir die Themen nach diesem Schema vorgestellt hatten, fragten wir die Jugendlichen, ob wir ihre Schwerpunkte erfasst hätten, ob wir etwas falsch verstanden hätten und ob Themen fehlten und wenn ja, welche das wären. Wurden fehlende Themen benannt oder Änderungen vorgeschlagen, so ergänzten wir dies auf Moderationskarten. Alle Themen, die von den Jugendlichen bestätigt wurden, schrieben wir ebenso auf Moderationskarten. Dann hängten wir alle Themenkarten an eine Pinnwand.

Nun forderten wir die Jugendlichen auf, verdeckt hinter der Pinnwand und nacheinander mit zehn Klebepunkten die wichtigsten Themen, bei denen sie eine Veränderung für möglich hielten, zu priorisieren. Wie viele Klebepunkte sie einem Thema geben wollten, blieb ihnen überlassen. 


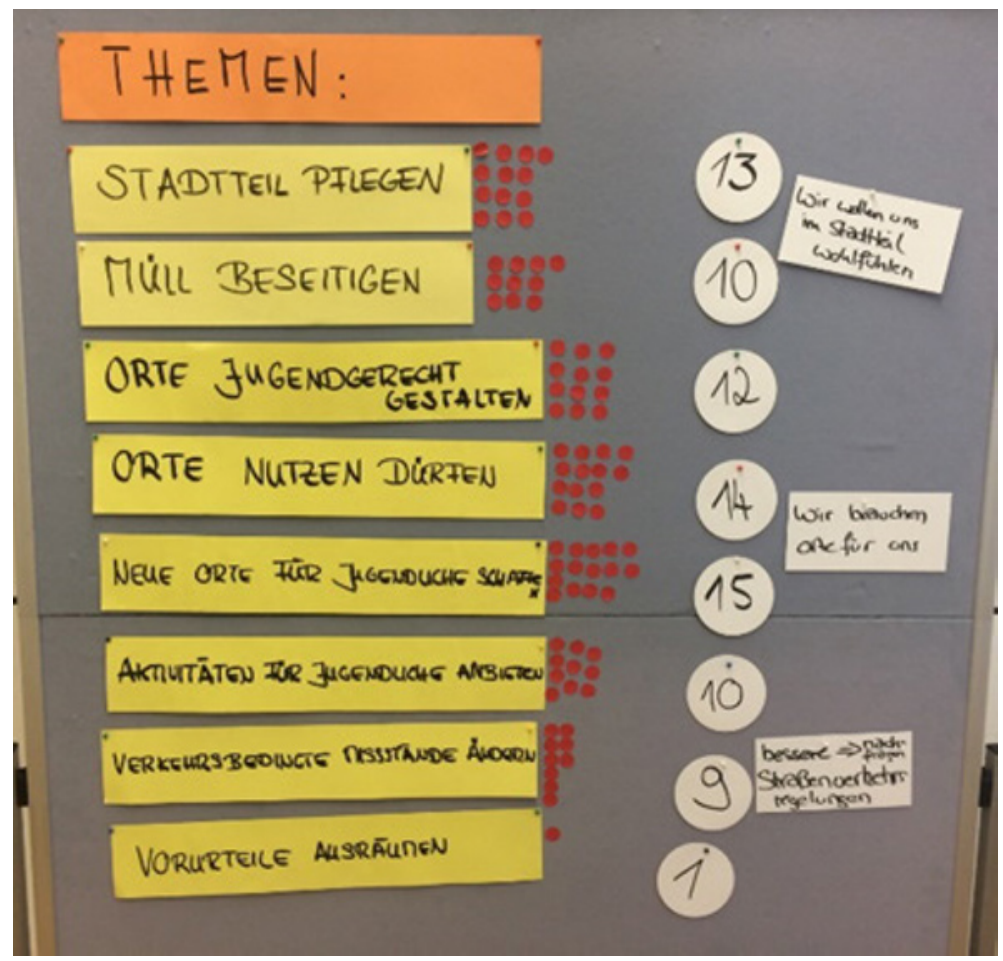

Abb. 4 Abstimmung über die Themen mittels Klebepunkten (im Auswertungsworkshop entstandenes Foto)

\section{Diskussion und Lokalisieren der Handlungsbedarfe}

Nachdem alle Jugendlichen ihre Punkte vergeben hatten, stimmten wir gemeinsam $\mathrm{ab}$, ob Themen mit niedrigeren Punktzahlen nicht weiter verfolgt werden sollten.

Wir erörterten nun nacheinander die übrig gebliebenen Themen anhand folgender Fragen:

- Was sollte getan werden?

- Wer kann hier etwas tun?

- Was können wir tun, damit es vorangeht? 
Wir lokalisierten, wo es um konkrete Orte ging, auf einer Stadtteilkarte die Handlungsbedarfe und -möglichkeiten. Zum Beispiel kennzeichneten wir mit einem roten Klebepunkt alle Orte, an denen der Müll regelmäßiger entsorgt werden sollte. Die Ergebnisse der nicht ortsgebundenen Themen protokollierten wir für die spätere Diskussion und Formulierung von Handlungsempfehlungen.

\section{Ausklang}

Beim Pizzaessen fassten wir das Ergebnis des Workshops zusammen und teilten den Jugendlichen mit, wie es weitergehen würde. Wir erinnerten an den nächsten Termin, der mit der Forschungsgemeinschaft stattfinden sollte.

\section{Beispiel für die Erarbeitung von Handlungsempfehlungen in der Forschungsgemeinschaft aus dem KEG}

Nach dem Auswertungsworkshop mit den Jugendlichen fanden zwei Treffen mit der gesamten Forschungsgemeinschaft statt. Dort wurden die Ergebnisse der Jugendlichen miteinander diskutiert sowie Handlungsempfehlungen und Lösungen erarbeitet. Vor dem ersten gemeinsamen Treffen gab es einen Termin, an dem die Ergebnisse der Jugendlichen der Forschungsgemeinschaft vorgestellt wurden. Dieses Treffen diente dazu, die Forschungsgemeinschaft an die ohne sie erfolgten Prozesse und an deren Ergebnisse anzuschließen und sie für die Perspektiven und Belange der Jugendlichen zu sensibilisieren.

Im ersten gemeinsamen Treffen arbeiteten wir in gemischten Kleingruppen (Akteur ${ }^{\star}$ innen, jugendliche Mitforschende, Vertreter ${ }^{\star}$ innen der Hochschule). Eine von den Wissenschaftler ${ }^{*}$ innen vorbereitete Ergebnisübersicht fungierte dabei als Diskussionseinstieg. Zwei Felder blieben zu Beginn der Treffen leer: Die Felder „Diskussionspunkte in der Forschungsgemeinschaft“ sowie „Handlungsempfehlungen/Lösungsvorschläge “ wurden von einer Protokollant ${ }^{\star}$ in damit gefüllt, was in den Treffen diskutiert und gemeinsam erarbeitet wurde.

Die Kleingruppen diskutierten die Themen der Jugendlichen und erarbeiteten Handlungsempfehlungen und Lösungsmöglichkeiten. Anschließend trugen sie ihre Ergebnisse im Plenum vor. Insgesamt bearbeiteten drei Gruppen je etwa sechs Themen in den zwei dreistündigen Treffen. 


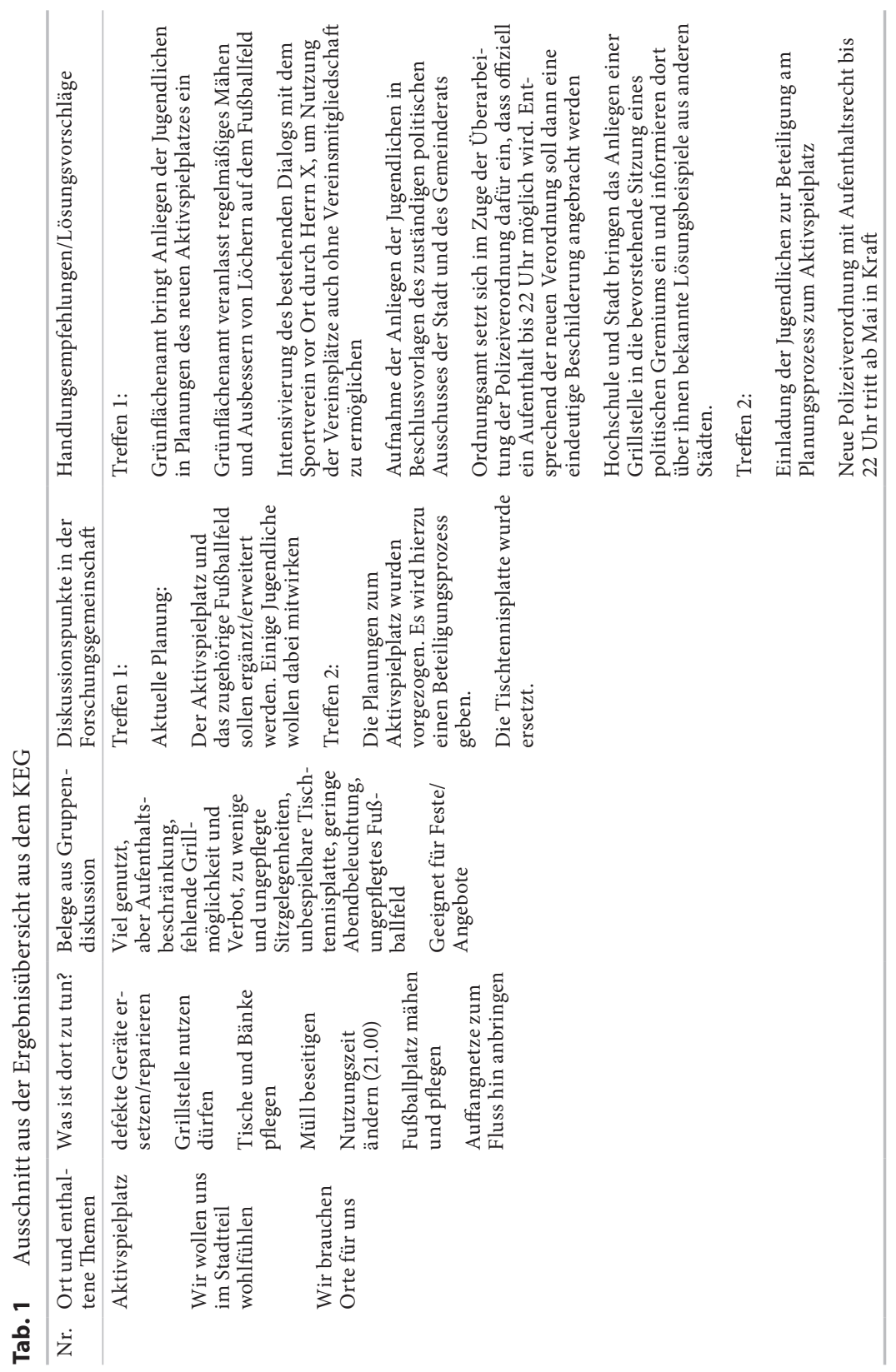




\section{Schritt 6 Die Ergebnisse veröffentlichen}

In diesem Schritt der Photovoice-Forschung präsentieren Sie die Ergebnisse und Handlungsempfehlungen einer breiteren Öffentlichkeit. Vor allem die entsprechenden Entscheidungsträgerinnen sollen für die Anliegen der Jugendlichen sensibilisiert werden und sich aktiv für die Umsetzung der Handlungsempfehlungen einsetzen. Überlegen Sie daher gemeinsam in der Forschungsgemeinschaft, auf welche Stellen Sie zugehen, wie Sie dies tun wollen und wer dabei welche Aufgabe(n) übernimmt. Wie breit Sie die Ergebnisse streuen und mit welchen Mitteln Sie das tun, ist abhängig von den Zielen Ihrer Photovoice-Forschung und dem Setting, in dem diese stattfindet.

Photovoice bietet durch die Arbeit mit Fotografien zahlreiche interessante Möglichkeiten, die Ergebnisse anschaulich und authentisch zu veröffentlichen. Viele Photovoice-Projekte veranstalten Ausstellungen mit den Fotos, die im Rahmen der Forschung entstanden sind. Sie können zum Beispiel auch Poster oder Plakate gestalten, um sie an ausgewählten Orten aufzuhängen, Kataloge erstellen, Aktionen veranstalten oder Berichte in der lokalen Presse veröffentlichen.

Im KEG produzierten wir gemeinsam einen Film, um die Themen der Jugendlichen anschaulich zu zeigen und ihnen eine Stimme zu geben. Er wurde bei nahezu jeder Ergebnispräsentation gezeigt. Sie können sich diesen unter folgendem Link ansehen: http://partkommplus.de/teilprojekte/keg/wie-stellen-sich-jugendliche-ihren-stadtteil-vor/.

Die Basis des Films bildeten die Fotos und Videos der Jugendlichen, die Filmaufnahmen aus der Schulung und die Tonbandaufnahmen aus den Gruppendiskussionen. Wir hatten die Jugendlichen in der Schulung gebeten, Statements abzugeben, warum sie im KEG mitmachen und was sie sich erhoffen - auch diese wurden im Film verwendet. Für die Erstellung des Storyboards - der Geschichte des Films - und für den Schnitt arbeiteten wir mit einem Experten zusammen.

Wir stellten die Ergebnisse der Photovoice-Forschung in verschiedener Form und vor unterschiedlichem Publikum vor: bei einer Sitzung eines stadtweiten politischen Gremiums und in einem politischen Gremium des Bezirks als Vortrag mit Diskussion, wo wir auch das erste Mal unseren Film öffentlich zeigten. Zusätzlich trafen sich drei der mitforschenden Jugendlichen mit dem Vorsitzenden dieses Bezirksgremiums, um intensiver miteinander ins Gespräch zu kommen und gemeinsam zu erörtern, wie die Interessen der Jugendlichen auch künftig weitergetragen werden können.

Auf einem Fest im Stadtteil gestalteten wir gemeinsam mit den mitforschenden Jugendlichen einen Stand, um mit Bewohner*innen über die Ergebnisse sowie über Anliegen und Interessen der Jugendlichen im Bezirk in einen Austausch zu kommen. Auch dort zeigten wir unseren Film sowie von den Jugendlichen entworfene Plakate. 
Die beteiligten Wissenschaftler*innen hielten zudem einen Vortrag mit anschlieBender Diskussion auf Bundesebene und, zusammen mit der Begleitperson, auf einem internationalen Kongress. Bei beiden Gelegenheiten zeigten wir wiederum den Film.

Darüber hinaus wurden das Projekt und dessen Ergebnisse in verschiedenen Printpublikationen vorgestellt.

Unter http://partkommplus.de/teilprojekte/keg/produkte/ finden Sie alle aus KEG entstandenen Veröffentlichungen.

\section{Schritt 7 Die Photovoice-Forschung evaluieren}

Die Evaluation ist der letzte Schritt einer Photovoice-Forschung. Zum einen überprüfen Sie mit der Evaluation, ob Ihr Projekt die gesteckten Ziele erreicht hat. Zum anderen können Sie durch die Evaluation beurteilen, was Ihre Photovoice-Forschung neben der Zielerreichung sonst noch bewirkt hat: bei den jugendlichen Mitforschenden, den beteiligten Akteur ${ }^{\star}$ innen, auf der politischen Ebene und im Forschungsfeld selbst. Die Evaluation kann zusätzlich auch prozessbegleitend erfolgen. Sich hier regelmäßig Rückmeldungen zum Forschungsvorgehen und dessen Zwischenergebnissen einzuholen, ermöglicht es, ggf. notwendige Anpassungen vorzunehmen, und stellt so sicher, dass sich die Durchführung des Projekts an den Anliegen und Interessen der Beteiligten orientiert.

In persönlichen Einzelgesprächen, in Gruppenfeedbacks oder durch Fragebögen kann während und am Ende des Forschungszeitraums gezielt Rückmeldung zum Vorgehen und zu den Wirkungen der Photovoice-Forschung eingeholt werden. Aber auch Gedächtnis- und Beobachtungsprotokolle von Treffen bilden eine gute Grundlage, um Ihre Photovoice-Forschung zu bewerten. Wie systematisch Sie bei der Evaluation vorgehen und was Sie dabei herausfinden möchten, ist abhängig von den zuvor gesetzten Zielen und davon, ob und wie Sie die Evaluationsergebnisse, beispielsweise für die Entwicklung von weiteren Projekten und Maßnahmen, nutzen wollen.

Im KEG war die Evaluation bereits in den Forschungsprozess eingebettet: Wir fragten die mitforschenden Jugendlichen in jedem Workshop, was sie während der jeweiligen Forschungsphase gut fanden, was eher nicht und was vielleicht auch schwierig war. Wir protokollierten die Rückmeldungen und passten unser weiteres Vorgehen an, wo es notwendig erschien. Diese Evaluation betraf die Gestaltung des Prozesses der Photovoice-Forschung.

Wir engagierten außerdem eine Sozialpädagogin, die gemeinsam mit den Jugendlichen deren Erfahrungen mit Photovoice gezielt reflektierte und diskutierte, was 
das Projekt aus Sicht der Jugendlichen gebracht hatte. Sie leitete die Jugendlichen an, aus den für sie wesentlichen Aspekten etwas Kreatives zu gestalten. Unsere Fragen für diese kreative Rückschau und Evaluation waren:

- Was haben wir gemeinsam erreicht?

- Was hat es euch gebracht, dabei zu sein?

- Wenn ihr morgen aufwacht und alles so ist, wie es sein soll, was hat sich dann in (Name des Bezirks) verändert?

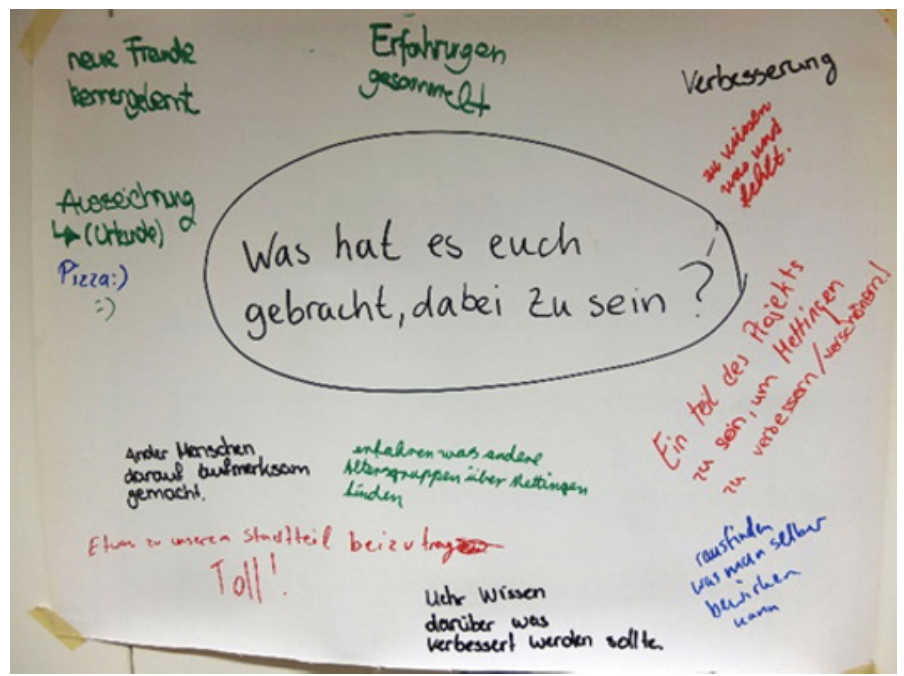

Abb. 5 Mindmap Erfahrungen mit Photovoice (in der Evaluation entstandenes Foto)

Auch von der Forschungsgemeinschaft holten wir bei jedem Treffen Feedback zum Vorgehen, zum aktuellen Stand und zu den (Zwischen-)Ergebnissen ein. Hier steuerten wir im Forschungsprozess, wenn notwendig, ebenfalls entsprechend nach. Die Forschungsgemeinschaft war zudem der institutionalisierte Ort, um gemeinsam mit den Jugendlichen deren Handlungsbedarfe zu besprechen, auszuhandeln, was möglich war, und Veränderungen einzuleiten oder umzusetzen. Damit war die Überprüfung eines wesentlichen Projektziels - ob Veränderungen im Sinne der Jugendlichen initiiert wurden - integraler Bestandteil der Arbeit der Forschungsgemeinschaft. 
Die Rückmeldungen der Beteiligten in politischen Gremien wurden von den Wissenschaftler*innen in Mitschriften oder (Gedächtnis-)Protokollen festgehalten, um Wirkungen auf der politischen Ebene erfassen zu können.

Alle gesammelten Rückmeldungen wurden neben der laufenden Auswertung von den Wissenschaftler*innen der Forschungsgemeinschaft am Ende des Projekts analysiert und im Beitrag 4 Photovoice als partizipative Methode: Wirkungen auf individueller, gemeinschaftlicher und gesellschaftlicher Ebene in diesem Buch verarbeitet.

\section{Anhang}

\section{Hilfreiche Seiten im Netz}

Auf dem YPARHUB finden Sie Übungseinheiten für die verschiedenen Phasen einer Photovoice-Forschung sowie zu vielen weiteren partizipativen Forschungsmethoden mit Jugendlichen. Die Seite ist in englischer Sprache: http://yparhub.berkeley.edu/.

Die englischsprachige Community Tool Box liefert gute Argumente für die Anwendung von Photovoice und bietet weiterführende Online-Ressourcen zur Methode: https://ctb.ku.edu/en.

Ein gut verständliches, allerdings englischsprachiges Video zum Vorgehen in einer Photovoice-Forschung finden Sie unter: https://www.youtube.com/watch?$\mathrm{v}=\mathrm{kAszQx62XxE \text {. }}$

Die Alice Salomon Hochschule Berlin entwickelte ein Video zum Vorgehen in Fokusgruppen. Es liefert auch für die Durchführung von Gruppendiskussionen in Photovoice-Projekten hilfreiche Anregungen: https://www.ash-berlin.eu/forschung/ forschungsprojekte-a-z/partnet-lernvideos-pilotstudie/.

Das Online-Magazin irights hat eine gut verständliche Beschreibung der Rechtslage zur Arbeit mit Fotografien publiziert: https://irights.info/artikel/auf-motivsuche $/ 5344$.

Auf den Seiten des kanadischen Wellesley Institute finden Sie eine dreiteilige englischsprachige Publikationsreihe, „Peer Research in Action I-III“ von Sarah Flicker, Adrian Guta und Brenda Roche, die sich intensiv mit den wesentlichen Aspekten der Arbeit mit Peer-Forschenden auseinandersetzt und daher auch für Photovoice-Projekte interessant ist. Die dritte Publikation befasst sich speziell mit ethischen Fragen, die partizipative Forschungen wie solche mit Photovoice aufwerfen: http://www.wellesleyinstitute.com/publications/peer-research-in-action/.

Im bereits erwähnten PaKoMi-Handbuch können Sie ausführlich nachlesen, wie sich innerhalb partizipativer Projekte zusammenarbeiten und forschen lässt. 
Es ist voll von Beispielen aus dem PaKoMi-Projekt: http://www.hiv-migration.de/ sites/default/files/publikationen/PaKoMi\%20Handbuch\%20Deutsch.pdf.

Auf der Homepage des Forschungsverbundes PartKommPlus finden Sie ausführliche Informationen zum Verbund und alle im KEG und in den anderen Projekten entstandenen Veröffentlichungen: http://partkommplus.de/.

\section{Zum vertiefenden Weiterlesen}

Wie partizipative Forschungsprojekte von A bis Z durchgeführt werden, ist anschaulich in folgendem Lehrbuch beschrieben: von Unger, Hella (2014). Partizipative Forschung: Einführung in die Forschungspraxis. Wiesbaden: Springer VS.

Eine gut verständliche und sehr praktische Einführung in die Praxisforschung bietet folgendes Lehrbuch: van der Donk, Cyrilla; van Lanen, Bas \& Wright, Michael T. (2014). Praxisforschung im Sozial- und Gesundheitswesen. Bern: Huber.

Dieser englischsprachige Sammelband behandelt alle möglichen Aspekte von Aktionsforschung - von der historischen Entwicklung bis zur konkreten Umsetzung: Reason, Peter \& Bradbury, Hilary (2013). The SAGE Handbook of Action Research: Participative Inquiry and Practice. 2. Aufl. London u. a.: SAGE.

\section{Verwendete und weiterführende Literatur}

Bergold, J. \& Thomas, S. (2012). Partizipative Forschungsmethoden: Ein methodischer Ansatz in Bewegung. Forum Qualitative Sozialforschung / Forum: Qualitative Social Research, 13(1), Art. 30. http://nbn-resolving.de/urn:nbn:de:0114-fqs1201302 [Zugriff: 14. August 2018].

Brandes, Sven \& Stark, Wolfgang (2016). Empowerment/Befähigung. In Bundeszentrale für gesundheitliche Aufklärung (BZgA) (Hrsg.), Leitbegriffe der Gesundheitsförderung und Prävention. https://www.leitbegriffe.bzga.de/alphabetisches-verzeichnis/empowermentbefaehigung/ [Zugriff: 09. Februar 2020].

Bundeszentrale für gesundheitliche Aufklärung (BZgA) (Hrsg.) (2018). Leitbegriffe der Gesundheitsförderung und Prävention. Glossar zu Konzepten, Strategien und Methoden. https://www.leitbegriffe.bzga.de/fileadmin/user_upload/leitbegriffe/e-Books/E-Book_Leitbegriffe_2018_08.pdf [Zugriff: 09. Februar 2020].

Deinet, Ulrich (2009) (Hrsg.). Methodenbuch Sozialraum. Wiesbaden: VS Verlag für Sozialwissenschaften.

Deinet, Ulrich (2009) (Hrsg.). Sozialräumliche Jugendarbeit: Grundlagen, Methoden und Praxiskonzepte. 3., überarb. Aufl. Wiesbaden: VS Verlag für Sozialwissenschaften.

Deinet, Ulrich (2009). Analyse- und Beteiligungsmethoden. In Ulrich Deinet (Hrsg.), Methodenbuch Sozialraum (S.65-86). Wiesbaden: VS Verlag für Sozialwissenschaften. 
Flick, Uwe; von Kardorff, Ernst \& Steinke, Ines (2015) (Hrsg.). Qualitative Forschung: Ein Handbuch. Hamburg: Rowohlt.

Glaser, Barney G. \& Strauss, Anselm L. (1998). Grounded Theory. Strategien qualitativer Forschung. Bern u. a.: Huber.

Krisch, Richard (2009). Methoden qualitativer Sozialraumanalyse als zentraler Baustein sozialräumlicher Konzeptentwicklung. In Ulrich Deinet (Hrsg.), Sozialräumliche Jugendarbeit: Grundlagen, Methoden und Praxiskonzepte (S. 161-173). 3., überarb. Aufl. Wiesbaden: VS Verlag für Sozialwissenschaften.

Kress, Bärbel \& Kern, Eva (2013). Soziometrische Aufstellung in Gruppen und Teams im Unternehmenskontext Personal- und Organisationsentwicklung im Stehen. In Christian Stadler (Hrsg.), Soziometrie: Messung, Darstellung, Analyse und Intervention in sozialen Beziehungen (S.213-235). Wiesbaden: Springer VS.

Mayring, Philipp (2015). Qualitative Inhaltsanalyse. In Uwe Flick, Ernst von Kardorff \& Ines Steinke (Hrsg.), Qualitative Forschung: Ein Handbuch (S. 468-475). Hamburg: Rowohlt. Mayring, Philipp (2016). Einführung in die qualitative Sozialforschung. 6., überarb. Aufl. Weinheim und Basel: Beltz.

Hartung, Susanne \& Rosenbrock, Rolf (2015). Settingansatz/Lebensweltansatz. In Bundeszentrale für gesundheitliche Aufklärung (BZgA) (Hrsg.), Leitbegriffe der Gesundheitsförderung und Prävention. doi:10.17623/BZGA:224-i106-1.0.

Stadler, Christian (2013) (Hrsg.). Soziometrie: Messung, Darstellung, Analyse und Intervention in sozialen Beziehungen. Wiesbaden: Springer VS.

von Unger, Hella \& Gangarova, Tanja (2011). PaKoMi Handbuch. HIV-Prävention für \& mit Migrant/inn/en. Berlin: Deutsche AIDS-Hilfe. https://www.hiv-migration.de/sites/default/ files/publikationen/PaKoMi\%20Handbuch\%20Deutsch.pdf [Zugriff: 26. Januar 2020].

Wang, Caroline C. (1999). Photovoice: a participatory action research strategy applied to women's health. Journal of women's health, 8(2), 185-192.

Wang, Caroline C. \& Burris, Mary A. (1997). Photovoice: concept, methodology, and use for participatory needs assessment. Health education \& behavior, 24(3), 369-387.

Open Access Dieses Kapitel wird unter der Creative Commons Namensnennung 4.0 International Lizenz (http://creativecommons.org/licenses/by/4.0/deed.de) veröffentlicht, welche die Nutzung, Vervielfältigung, Bearbeitung, Verbreitung und Wiedergabe in jeglichem Medium und Format erlaubt, sofern Sie den/die ursprünglichen Autor(en) und die Quelle ordnungsgemäß nennen, einen Link zur Creative Commons Lizenz beifügen und angeben, ob Änderungen vorgenommen wurden.

Die in diesem Kapitel enthaltenen Bilder und sonstiges Drittmaterial unterliegen ebenfalls der genannten Creative Commons Lizenz, sofern sich aus der Abbildungslegende nichts anderes ergibt. Sofern das betreffende Material nicht unter der genannten Creative Commons Lizenz steht und die betreffende Handlung nicht nach gesetzlichen Vorschriften erlaubt ist, ist für die oben aufgeführten Weiterverwendungen des Materials die Einwilligung des jeweiligen Rechteinhabers einzuholen.

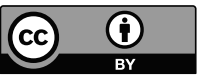

